\title{
Urinary tract infection caused by nontypable Haemophilus influenzae in the elderly
}

Since the introduction of the Haemophilus influenzae serotype $\mathrm{b}(\mathrm{Hib})$ vaccine, the decrease in invasive Hib disease predominantly affecting children and the relative increase in nontypable $H$. influenzae (NTHi) infections have been well documented in many parts of the world. The increasing trend in the number of cases, especially among older adults, deserves attention (Heath et al., 2001; De Almeida et al., 2005; Dworkin et al., 2007; Tsang et al., 2007).

$H$. influenzae has been rarely implicated as the causative agent of urinary tract infections in adults. The isolation of $H$. influenzae from a urine sample was first reported in 1898 when Kretz recovered influenza bacillus from the urine sample of a 36-year-old man with haematuria and polyuria (Wright, 1905). Since then, fewer than 30 cases of this bacterium as the cause of adult urinary tract infections have been reported (Reichman, 1999; Papaioannides et al., 2002). In Sweden, a study over a 24-year period showed 36 children with Haemophilus species infection (Hansson et al., 2007).

We report a rare case of NTHi urinary infection in an 87-year-old man and discuss the possible underestimation of the true incidence of $H$. influenzae in urinary tract infections. The patient was admitted to an emergency hospital with aorto-iliac-femoral artery disease and renal artery stenosis. Twenty-four hours after surgical angioplasty, the patient showed a temperature of $38{ }^{\circ} \mathrm{C}$. Urine was collected for urinary sediment analysis and culture. At the hospital laboratory, the urine was routinely cultured on cysteine-lactose-electrolyte-deficient (CLED) agar (Oxoid). After inoculation, the urine sample was stored under refrigeration $\left(4-8{ }^{\circ} \mathrm{C}\right)$. Microscopic examination and Gram stain of a clean voided urine specimen revealed 30-40 white blood cells per oil immersion field and the presence of Gram-negative coccobacilli. In view of these findings, a urine sample was cultured on brain heart infusion chocolate agar (Difco) enriched with $10 \%$ defibrinated rabbit blood at $37{ }^{\circ} \mathrm{C}$ for $24 \mathrm{~h}$, and bacterial growth was detected after $24 \mathrm{~h}$. Gram staining of bacterial colonies was performed, showing abundant pleomorphic Gram-negative rods suggesting Haemophilus species. The culture was sent to the National Institute for Quality Control in Health - FIOCRUZ (INCQS) for additional identification and typing tests such as slide agglutination for serotype determination, biotyping, antimicrobial sensitivity and PCR capsular typing as previously described (Falla et al., 1994; Campos, 1999). Urine culture collected after surgery inoculated on CLED agar was negative after $48 \mathrm{~h}$. Treatment was initiated with i.v. $3 \mathrm{~g} / 6 \mathrm{~h}$ ampicillin-sulbactam ( $1 \mathrm{~g}$ sulbactam $+2 \mathrm{~g}$ ampicillin) for 8 days, since the initial suspected aetiological agent of the infection was $H$. influenzae. After culture confirmation, the antibiotic therapy was parenteral $8 \mathrm{~g} / 1 \mathrm{~g}$ per day $(4 \mathrm{~g} / 500 \mathrm{mg} /$ $12 \mathrm{~h}$ ) for 8 days of piperacillintazobactam (Wagenlehner et al., 2003). Negative slide agglutination for serotyping, biotyping and negative PCR capsular typing confirmed NTHi biotype VIII as the causative agent of infection. The NTHi strain isolated was nalidixic acid resistant $\left(\mathrm{MIC}>256 \mu \mathrm{g} \mathrm{ml}^{-1}\right.$ ), $\beta$-lactamase-negative, and sensitive to ampicillin and fluoroquinolones (ciprofloxacin, levofloxacin, lemofloxacin, moxifloxacin). After antibiotic therapy, the patient showed complete resolution of symptoms, return of normal function and normalization of blood parameters.

There are few reports of urinary tract infection caused by Haemophilus species. The inability of Haemophilus species to grow in standard media commonly used for culture of uropathogens suggests that the true frequency of these strains as a cause of urinary tract infections is underestimated. H. influenzae has rarely been implicated as the causative agent in urinary tract infections in adults, with most cases afflicting men with anatomical or functional genito-urinary abnormalities. The true incidence of $H$. influenzae genito-urinary infection is unknown. The apparent rarity of $H$. influenzae urinary tract infection may be ascribed to three factors: (i) bacteriological media for recovery of uropathogens do not support the growth of $H$. influenzae; (ii) the organism is not generally part of the genitoperineal flora; and (iii) growth of $H$. influenzae is inhibited by urine even when it is supplemented with the necessary growth factors (Gabre-Kidan et al., 1984).

$H$. influenzae may be a more common uropathogen in adults than previously recognized. We should consider the use of more sensitive culture techniques and greater physician awareness of the genitourinary pathogenicity of this bacterium, particularly in male elderly patients with anatomical or functional genito-urinary abnormalities, to enhance the detection of $H$. influenzae urinary tract infection. Therefore the nalidixic acid resistance of $H$. influenzae should be investigated according to the age of patients (PérezVázquez et al., 2004). Prompt institution of therapy with antibiotics active against $H$. influenzae following sensitivity studies could prevent systemic septic complications in this group of frail patients.

Clearly, further studies are required to characterize the impact on pathogenicity and virulence of NTHi in unimmunized adult patients and the epidemiology of such micro-organisms. Also, nalidixic acid resistance may be useful as a baseline for future studies to monitor antimicrobial susceptibilities including susceptibility to fluoroquinolones.

To our knowledge, this is the first report of urinary tract infection caused by NTHi in adults in Brazil after the introduction of the Hib conjugate vaccine. 


\section{Acknowledgements}

This study was supported by CAPES/CNPq/ INCQS/FIOCRUZ/MS.

\section{Antonio Eugenio Castro Cardoso de Almeida, ${ }^{1}$ Nathalia Gonçalves Santos Caldeira, ${ }^{1}$ Letícia Ferreira Lima Schroeder, ${ }^{1}$ Alice Aurora Batalha de Jesus, ${ }^{1}$ Lucia Rodrigues Serradas, ${ }^{2}$ Andrea Ferreira da Costa, ${ }^{2}$ Carla Ormundo Gonçalves Ximenes Lima ${ }^{2}$ and Ivano de Filippis ${ }^{1}$}

${ }^{1}$ Instituto Nacional de Controle da Qualidade em Saúde (INCOS), Departamento de Microbiologia, Fundação Oswaldo Cruz (FIOCRUZ), Av. Brasil, 4365 - Manguinhos, 21045-900 Rio de Janeiro, Brazil

${ }^{2}$ Hospital de Clínicas RioMar Barra, Av. Cândido Portinari, 555 - Barra, 22793310 Rio de Janeiro, Brazil

Correspondence: Antonio Eugenio Castro Cardoso de Almeida (eugenio.almeida@incqs.fiocruz.br)

Campos, J. M. (1999). Haemophilus. In Manual of Clinical Microbiology, 7th edn, pp. 604-613. Washington, DC: American Society for Microbiology.
De Almeida, A. E. C. C., de Filippis, I., de Abreu, A. O., Ferreira, D. G., Gemal, A. L. \& Marzochi, K. B. F. (2005). Occurrence of Haemophilus influenzae strains in three Brazilian states since the introduction of a conjugate Haemophilus influenzae type b vaccine. Braz J Med Biol Res 38, 777-781.

Dworkin, M. S., Park, L. \& Borchardt, S. M. (2007). The changing epidemiology of invasive Haemophilus influenzae disease, especially in persons $>65$ years old. Clin Infect Dis 44, 810 816.

Falla, T. J., Crook, D. W. M., Brophy, L. N., Maskell, D., Kroll, J. S. \& Moxon, E. R. (1994). PCR for capsular typing of Haemophilus influenzae. J Clin Microbiol 32, 2382-2386.

Gabre-Kidan, T., Lipsky, B. A. \& Plorde, J. J. (1984). Hemophilus influenzae as a cause of urinary tract infections in men. Arch Intern Med 144, 1623-1627.

Hansson, S., Svedhem, A., Wennerström, M. \& Jodal, U. (2007). Urinary tract infection caused by Haemophilus influenzae and Haemophilus parainfluenzae in children. Pediatr Nephrol 22, 1321-1325.

Heath, P. T., Booy, R., Azzopardi, H. J., Slack, M. P. E. F., Fogarty, J. F., Moloney, A. C. F., Ramsay, M. E. M. \& Moxon, E. R. F. (2001). Non-type b Haemophilus influenzae disease: clinical and epidemiologic characteristics in the Haemophilus influenzae type b vaccine era. Pediatr Infect Dis J 20, 300305.

Papaioannides, D., Bouropoulos, C., Sinapides, D. \& Akritidis, N. (2002).

Haemophilus influenzae acute pyelonephritis in the elderly. Int Urol Nephrol 34, 23-24.

Pérez-Vázquez, M., Román, F., Aracil, B., Cantón, R. \& Campos, J. (2004). Laboratory detection of Haemophilus influenzae with decreased susceptibility to nalidixic acid, ciprofloxacin, levofloxacin, and moxifloxacin due to $g y r A$ and parC mutations. J Clin Microbiol 42, 1185-1191.

Reichman, J. M. (1999). Haemophilus influenzae pyelonephritis in adults. Arch Intern Med 159, 316.

Tsang, R. S. W., Sill, M. L., Skinner, S. J., Law, D. K. S., Zhou, J. \& Wylie, J. (2007).

Characterization of invasive Haemophilus influenzae disease in Manitoba, Canada, 20002006: invasive disease due to non-type $b$ strains. Clin Infect Dis 44, 1611-1614.

Wagenlehner, E., Niemetz, A. \& Naber, G. (2003). Spectrum of pathogens and resistance to antibiotics in urinary tract infections and the consequences for antibiotic treatment: study of urology inpatients with urinary tract infections (1994-2001). Urologe A 42, 13-25.

Wright, J. H. (1905). An observation of the occurrence of the bacillus of influenza (Bacterium influenzae) in pyelo-nephrosis. Boston Med Surg J 152, 496. 